\title{
Biodegradation of recalcitrant compounds and phthalates by a consortium of Liometopum apiculatum gut microbiota
}

Jorge L. González-Escobar

Instituto Potosino de Investigacion Cientifica y Tecnologica

Marco A. Pereyra-Camacho

Instituto Potosino de Investigacion Cientifica y Tecnologica

Antonio De Léon-Rodríguez

Instituto Potosino de Investigacion Cientifica y Tecnologica

Alicia Grajales-Lagunes

Universidad Autonoma de San Luis Potosi

Antonio Reyes-Agüero

Universidad Autonoma de San Luis Potosi

Alicia Chagolla-López

Centro de Investigacion y de Estudios Avanzados Unidad Irapuato

Ana Paulina Barba de la Rosa ( $\nabla$ apbarba@ipicyt.edu.mx )

Instituto Potosino de Investigación Científica y Tecnológica https://orcid.org/0000-0003-4999-036X

Research article

Keywords: ant microbiota; cellulases-degrading activity; GC-MS; ligninases-degrading activity; MALDIBiotyping; phthalates-degrading activity

Posted Date: November 28th, 2019

DOI: https://doi.org/10.21203/rs.2.16926/v2

License: (1) (i) This work is licensed under a Creative Commons Attribution 4.0 International License. Read Full License 
The authors have withdrawn this preprint from Research Square 